\title{
Comparison of different triolein-based composite semipermeable membranes for passive sampling of organochlorine pesticides
}

\author{
TANG JianFeng, XU YiPing, MA Mei \& WANG ZiJian* \\ State Key Laboratory of Environmental Aquatic Chemistry, Research Center for Eco-Environmental Sciences, Chinese Academy of Sciences, \\ Beijing 100085, China
}

Received September 26, 2011; accepted November 11, 2011; published online March 12, 2012

\begin{abstract}
Passive sampling methods have been widely used for more than 20 years because they can provide information on time-weighted average concentrations $\left(C_{\mathrm{TWA}}\right)$ of hydrophobic organic contaminants in water. Many factors affect the efficiency and applicability of a passive sampling process. Among others, selection of the sampler design and choice of the supporting membrane material are important factors that determine the performance of passive samplers. In this work, the influences of some critical properties of membranes, such as membrane density, hydrophilicity of the membrane surface, and chemical and mechanical resistances, on the uptake of organic chlorinated pesticides (OCPs) were evaluated. Four membrane materials, namely cellulose acetate (CA), cellulose acetate butyrate (CAB), a cellulose acetate-cellulose triacetate blend (CA-CTA), and cellulose triacetate (CTA), and their triolein-embedded samplers were selected for the study. The four membranes are all hydrophilic; CAB has the highest contact angle and CA has the lowest. CTA is chemically and mechanically resistant, and has better tensile strength than the other membranes have. $\mathrm{CAB}$ does not have good chemical resistance and dissolves in hexane/dichloromethane solvent. CA and CTA alone showed lower uptake rates for the selected OCPs than with triolein-embedded passive samplers, indicating that triolein increased the accumulation capacity. Triolein-embedded CA (TECAM), CA-CTA (TECA-CTA), CTA (TECTA), and CAB (TECAB) as passive samplers showed similar uptake patterns; TECTA showed the highest uptake rates and TECAB showed the poorest accumulation. In summary, TECTA gave the best performance among all the tested composite semipermeable membranes.
\end{abstract}

passive sampler, triolein-embedded semipermeable membranes, organochlorine pesticides

Citation: Tang J F, Xu Y P, Ma M, et al. Comparison of different triolein-based composite semipermeable membranes for passive sampling of organochlorine pesticides. Chin Sci Bull, 2012, 57: 1788-1795, doi: 10.1007/s11434-012-5044-z

Passive sampling devices made of different materials have been widely used in environmental monitoring, especially for hydrophobic organic compounds. Their advantages include reliability, cost-effectiveness, and ease of application. Because they integrate concentrations over a certain exposure time, they can provide information on time-weighted average concentrations $\left(C_{\mathrm{TWA}}\right)$. Recently, various types of passive sampling devices and set-ups have been developed for monitoring organic pollutants in aquatic environments. These include lipid-filled semipermeable membrane devices (SPMDs) [1], Chemcatcher [2], membrane-enclosed sorptive coatings [3], and polar organic chemical integrative

\footnotetext{
*Corresponding author (email: wangzj@rcees.ac.cn)
}

samplers [4]. Their different designs and performances have recently been reviewed [5-7].

However, the range of possibilities for the development of new devices is restricted by the theoretical principles governing passive sampling [8]. It is clear that the ideal passive sampling device would be one which would allow high sampling rates and would be easy to apply. Passive samplers typically consist of two parts: the receiving phase that acts as a sink for the compounds under study, and, usually, a semipermeable membrane as a barrier through which the target compounds can permeate and diffuse. Sometimes in passive samplers without a sorbent the membrane is also the receiving phase. Uptake of a chemical from the environment is by passive diffusion; the mass of a contaminant 
accumulated is determined by its concentration in the environment, the length of exposure, and the sampling rate of the passive sampler. The sampling rate is determined by a number of factors, including environmental variables (temperature, water turbulence, and biofouling), the properties of the chemical, and the properties of the diffusion-limiting layer. Selection of the sampler design and the choice of membrane materials are therefore important factors that determine the performance of passive samplers $[9,10]$. Analyte diffusion coefficients in polymeric membranes and the sampler-water partition coefficients affect sampler behavior and analyte-sampling rates [11]. It has been reported that uptake rates of organochlorine pesticides on solvent-filled cellulose were lower by two orders of magnitude than those on polyethylene membranes [12]. Similarly, several different polymeric membranes have been tested for construction of Chemcatcher samplers. Because of their high degree of physical strength and good antifouling properties, polysulfone and poly(ether sulfone) membranes were selected for samplers designed to sample polar organic pollutants, and cellulose acetate (CA) was found to be suitable for inorganic ions [13]. Membranes play a decisive role in passive samplers; not only the sampling rates but also the flow sensitivities of passive samplers are related to the membrane materials [14]. When the membrane creates a barrier sufficient for avoiding the influence of changes in flow turbulence, the transfer can be limited by the membrane materials; on the other hand, transfer can be limited by the water boundary layer, thus allowing high sampling rates but increasing the sensitivity to turbulence.

SPMDs are currently the most widely used type of passive samplers for measuring pollutants in aquatic environments because of their high sensitivity and bioavailability. An SPMD consists of lay-flat polyethylene tubing containing a small amount of the neutral lipid triolein. Common disadvantage of SPMDs and most other passive samplers are laborious recovery of analytes from the samplers and the need for additional clean-up before analysis. Low-cost and simple pretreatment sample processing is required. Many efforts have been made to improve the configurations of passive samplers. Single-phase polymeric materials such as low-density polyethylene (LDPE) are used as passive samplers [11], and many different membrane materials have been assessed for their suitability as passive samplers [10,11]. We previously developed the use of triolein-embedded CA membrane (TECAM) passive sampling devices, and the results from both laboratory and field experiments showed that TECAMs can quickly and efficiently accumulate hydrophobic organic chlorinated pesticides (OCPs) from water [15]. TECAM also shows higher uptake rates of polycyclic aromatic hydrocarbons (PAHs) than SPMDs do [16]. The unique configuration of TECAM provides a larger ratio of membrane surface area to lipid volume and simpler pretreatment procedures. Another passive sampling device, called a TCAPE, consists of a thin film of triolein enclosed in a thin-walled tube made of a composite CA membrane supported by linear LDPE; the device is formed with an LDPE support and an external CA side; and it accumulates hydrophobic OCPs in water more quickly and efficiently than SPMDs do [17]. The results using TECAM and TCAPE both suggested that replacing LDPE with CA gives higher uptake rates of hydrophobic chemicals. However, whether CA could be used as a passive sampler, as LDPE can, is not certain. CA has poor chemical and mechanical resistances and fast biodegradability. A membrane with stronger mechanical and thermal performances is needed.

The objective of this study is to optimize the design of triolein-embedded composite semipermeable membranes using as passive sampler for hydrophobic substances. We evaluated the organic contaminant uptakes, physical strengths, membrane hydrophilicity, and solvent compatibilities of several candidate porous polymeric membranes. A singlephase polymeric membrane and triolein-embedded passive sampler were also compared to determine whether these polymeric membranes would be suitable as passive samplers.

\section{Materials and methods}

\subsection{Reagents and materials}

Standards of six selected OCPs, namely $\gamma$-hexachlorocyclohexane $(\gamma-\mathrm{HCH}), \beta$-hexachlorocyclohexane $(\beta$-HCH $)$, aldrin, heptachlor epoxide, 1,1-bis(p-chlorophenyl)-2,2-dichloroethylene ( $p, p^{\prime}$-DDE), and endrin, were purchased from Supelco (Bellefonte, PA, USA). Triolein of 97\% purity, CA (39.8 wt $\%, \mathrm{MW}=100000)$, cellulose acetate butyrate $(\mathrm{CAB})$ (17 wt $\%$ butyryl content, $\mathrm{MW}=65000)$, cellulose triacetate (CTA) (43.4 wt\% acetyl content), and maleic acid were purchased from Sigma-Aldrich (St. Louis, MO, USA). All organic solvents were HPLC or pesticide grade and obtained from Fisher Scientific (Fair Lawn, NJ, USA) and J. T. Baker (Phillipsburg, NJ, USA). All other chemicals were of analytical grade or above.

\subsection{Preparation of composite semipermeable mem- branes}

Different membrane-casting solutions and film-casting conditions, based on phase-inversion methods [18-20], are given in detail in Tables 1 and 2. Homogeneous solutions were prepared by ultrasonic oscillation for $30 \mathrm{~min}$ and shaking at $25^{\circ} \mathrm{C}$ overnight. Solutions were cast on a glass plate in layers of thickness $0.15 \mathrm{~mm}$. After solvent evaporation, the glass plate with the cast layer was immediately immersed in deionized water for at least $10 \mathrm{~min}$ to ensure complete phase separation, and then rinsed with deionized water. The triolein-embedded passive samplers were made by adding $1.5 \mathrm{wt} \%$ triolein to the casting solutions. Simple membranes without any added triolein were also prepared at the same time. All of the membranes were kept in deionized 
Table 1 Casting solutions used for production of four composite semipermeable membranes

\begin{tabular}{|c|c|c|c|c|c|c|}
\hline Membrane type & Polymer 1 & Polymer 2 & Solvent 1 & Solvent 2 & Additive 1 & Additive 2 \\
\hline $\mathrm{CA}$ & CA, $18 \%$ & & Acetone, $70 \%$ & Dioxane, $10 \%$ & $\mathrm{Mg}\left(\mathrm{ClO}_{4}\right)_{2} \cdot x \mathrm{H}_{2} \mathrm{O}, 2.0 \%$ & \\
\hline $\mathrm{CA} / \mathrm{CTA}$ & $\mathrm{CA}, 7 \%$ & CTA, 7\% & Dioxane, $47.5 \%$ & Acetone, $28.5 \%$ & Maleic acid, 3\% & Methanol, 7\% \\
\hline CTA & CTA, $13 \%$ & & Dioxane, $59 \%$ & Acetone, $19.6 \%$ & Maleic acid, $4.8 \%$ & Methanol, 3.6\% \\
\hline $\mathrm{CAB}$ & $\mathrm{CAB}, 14 \%$ & & Acetone, $79 \%$ & & $\mathrm{Mg}\left(\mathrm{ClO}_{4}\right)_{2} \cdot x \mathrm{H}_{2} \mathrm{O}, 3.5 \%$ & Water, $3.5 \%$ \\
\hline
\end{tabular}

Table 2 Film-casting conditions

\begin{tabular}{lccc}
\hline $\begin{array}{c}\text { Membrane } \\
\text { type }\end{array}$ & $\begin{array}{c}\text { Solvent } \\
\text { evaporation time }\end{array}$ & Temperature & Gelation medium \\
\hline $\mathrm{CA}$ & $30 \mathrm{~s}$ & $25^{\circ} \mathrm{C}$ & Water at room temperature \\
$\mathrm{CA} / \mathrm{CTA}$ & $30 \mathrm{~s}$ & $25^{\circ} \mathrm{C}$ & Ice water \\
$\mathrm{CTA}$ & $30 \mathrm{~s}$ & $25^{\circ} \mathrm{C}$ & Ice water \\
$\mathrm{CAB}$ & $60 \mathrm{~s}$ & $25^{\circ} \mathrm{C}$ & Ice water \\
\hline
\end{tabular}

water before use. The membranes were cut into small pieces $(4 \mathrm{~cm} \times 6 \mathrm{~cm})$ for use as passive samplers.

\subsection{Membrane characterization}

Membrane thickness: The membrane thickness was measured directly using a micrometer. The measurements were carried out at five different positions on the membrane.

Water content and porosity: The membranes kept in deionized water were wiped with clean paper tissue and the wet weights $W_{\mathrm{w}}$ were measured. The membranes were then kept in an oven at $60^{\circ} \mathrm{C}$ for $3 \mathrm{~h}$; the dry weights $W_{\mathrm{d}}$ were also measured. Porosity was calculated using the following formula:

$$
P_{\mathrm{r}}=\frac{W_{\mathrm{W}}+W_{\mathrm{d}}}{s \times d \times \rho} \times 100 \%,
$$

where $W_{\mathrm{w}}$ is the wet weight of the membrane $(\mathrm{g}), W_{\mathrm{d}}$ is the dry weight of the membrane, $s$ is the membrane area $\left(\mathrm{cm}^{2}\right)$, $d$ is the membrane thickness $(\mathrm{cm})$, and $\rho$ is the density of water $\left(\mathrm{g} / \mathrm{cm}^{3}\right)$.

Contact angle measurements: The hydrophilicity of the surface membranes were studied by contact angle measurements. The contact angle was measured using a contact angle goniometer. A drop of deionized water was put on the surface with a micro-syringe and observed using a camera. Each contact angle of the membrane was measured at least 10 times and the average value was used.

Mechanical tests: The mechanical properties of the membranes were tested using a tensile instrument $(\mathrm{BH}-$ 0908). Membranes of dimensions $10 \mathrm{~mm} \times 40 \mathrm{~mm}$ were prepared. The crosshead speed was set at $12.5 \mathrm{~mm} / \mathrm{min}$. The measurements were made at least 10 times and the average value was used.

Stability in solvents: To test the solvent stabilities of the selected membranes, hexane and dichloromethane (DCM), which were used in the TECAM extractions, were selected as the test solvents. Membrane sheets $(4 \mathrm{~cm} \times 6 \mathrm{~cm})$ were soaked in $100 \mathrm{~mL}$ of solvent and shaken for $30 \mathrm{~min}$. The solvent-polymer compatibilities were then observed.

\subsection{Uptake experiments and sample preparation}

Batch experiments were carried out using the previously described negligible depletion sampling method [21]. Briefly, $1 \mathrm{~L}$ of each solution was transferred to $1-\mathrm{L}$ conical flasks and spiked with the six test OCPs to give a nominal concentration of $500 \mathrm{ng} / \mathrm{L}$ for each analyte. One membrane was introduced into each flask, and the flasks were shaken at $150 \mathrm{r} / \mathrm{min}$ at $25^{\circ} \mathrm{C}$. The membranes were removed after exposure for $1 \mathrm{~h}$ and placed in a new flask filled with $1 \mathrm{~L}$ of test solution. The OCPs accumulated in the membranes were monitored throughout $10 \mathrm{~h}$ of exposure. All the experiments were carried out in triplicate.

Prior to extraction, the membranes were rinsed with deionized water and wiped with clean paper tissue. Each membrane $(4 \mathrm{~cm} \times 6 \mathrm{~cm})$ was ultrasonically extracted three times with $5 \mathrm{~mL}$ of hexane/DCM $(1: 1, \mathrm{v} / \mathrm{v})$ solvent for 10 -min cycles. After extraction, the solutions were combined and concentrated to $0.5 \mathrm{~mL}$ under a gentle stream of nitrogen, and solvent exchanged into $n$-hexane. An appropriate amount of internal standard (pentachloronitrobenzene) was added to the final concentrated extract prior to instrumental analysis.

\subsection{Instrumentation}

OCPs analyses were performed using an Agilent 6890 series gas chromatograph (GC) equipped with a 7863 autosampler and a micro-electron capture detector (ECD, Agilent Technologies, Palo Alto, CA, USA). A 30-m HP-1701 column $(30 \mathrm{~m} \times 0.25 \mathrm{~mm} \times 0.25 \mu \mathrm{m}$ film thicknesses, Agilent Technologies) was used to separate the analytes. Nitrogen was used as a carrier gas at a rate of $0.9 \mathrm{~mL} / \mathrm{min}$ under the constant flow mode. A 1- $\mu \mathrm{L}$ sample was injected into the GC using the splitless mode. The oven was programmed from 60 to $160^{\circ} \mathrm{C}$ (2-min hold time) at a rate of $20^{\circ} \mathrm{C} / \mathrm{min}$, and then to $260^{\circ} \mathrm{C}$ at a rate of $4^{\circ} \mathrm{C} / \mathrm{min}$ and holding for $5 \mathrm{~min}$. The injector and detector temperatures were maintained at $200^{\circ} \mathrm{C}$ and $280^{\circ} \mathrm{C}$, respectively. Qualitative identifications 
were made using retention time comparisons and the retention window was set at $0.5 \%$. Calibration curves were based on areas, using internal standards.

\subsection{Data analysis}

Passive sampling theory has been described in previous studies [21]. Briefly, if the amount of a chemical extracted by a passive sampler during a measurement is very small compared to the total amount in solution, the uptake of the chemical by the passive sampler can be simply described by a first-order, one-compartment model:

$$
C_{\mathrm{s}}=C_{\mathrm{w}} \frac{k_{\mathrm{u}}}{k_{\mathrm{e}}}\left(1-\mathrm{e}^{-k_{\mathrm{e}} t}\right) .
$$

where $t$ is the exposure time for the passive sampler, $C_{\mathrm{s}}$ is the concentrations of the target compound in the sampler at time $t, C_{\mathrm{w}}$ is the concentration of the analyte in water, $k_{\mathrm{u}}$ is the uptake rate-constant, and $k_{\mathrm{e}}$ is the overall elimination rate-constant.

When $k_{\mathrm{e}} t$ is small (i.e., $k_{\mathrm{e}} t \ll 1$ ), chemical uptake is linear and integrative, and eq. (2) reduces to

$$
C_{\mathrm{s}}=C_{\mathrm{w}} k_{\mathrm{u}} t
$$

When $k_{\mathrm{e}} t \gg 1$, eq. (2) reduces to an equilibrium model:

$$
K_{\text {passive sampler }}=\frac{C_{\mathrm{s}}}{C_{\mathrm{w}}}=\frac{k_{\mathrm{u}}}{k_{\mathrm{e}}},
$$

with $K_{\text {passive sampler }}$ being the steady-state passive-water partition coefficient. The chemical uptake into the passive sampler remains linear and integrative until the ratio of $C_{\mathrm{s}} / C_{\mathrm{w}}$ reaches approximately half-saturation, according to Huckins et al. [14].

\section{Results and discussion}

\subsection{Characterization of polymer membranes}

Some significant differences were found between the physicochemical and mechanical properties of the selected polymeric membranes. The results are summarized in Table 3.

\subsection{Mechanical properties and stabilities of polymer membranes}

The thicknesses of the membranes varied from 59.7 to $99.3 \mu \mathrm{m}$. Table 3 shows the data for the membranes prepared for this study; the data show that $\mathrm{CAB}$ had the highest thickness and CA had the lowest thickness.

Membrane thickness is an important factor for semipermeable membranes in passive samplers used in aquatic environments. Thin polymer membranes often lack mechanical stability, especially when the membrane is used in highly turbulent aquatic environments. Membrane thickness is also important in determining the uptake rate-constants of hydrophilic organic compounds [14]; when compounds are under water boundary layer control, higher sampling rates can be obtained when the transport resistance (membrane thickness) in the polymer membrane is small compared to that of the water boundary layer [11]. Thin polymer membranes seem to have an advantage over thicker ones. Our results showed that the thicknesses of all the selected polymer membranes were less than $100 \mu \mathrm{m}$; these are thinner than other passive samplers such as PDMS (150-200 $\mu \mathrm{m})$ and are similar to LDPE (about $60 \mu \mathrm{m}$ ).

Mechanical properties are considered to be critical factors for membrane passive samplers used in aquatic environments because high turbulence or flow could damage the membranes. The mechanical property parameters (tensile

\begin{tabular}{|c|c|c|c|c|c|c|c|c|}
\hline Property & CA & TECAM & CA-CTA & TECA-CTA & CTA & TECTA & $\mathrm{CAB}$ & TECAB \\
\hline Thickness $(\mu \mathrm{m})$ & $59.7 \pm 11.4$ & $67.5 \pm 9.3$ & $75.0 \pm 4.5$ & $70.3 \pm 7.2$ & $81.6 \pm 7.3$ & $68.4 \pm 6.9$ & $99.3 \pm 17.2$ & $79.6 \pm 4.9$ \\
\hline Mass (g/sampler) & $0.137 \pm 0.012$ & $0.148 \pm 0.023$ & $0.181 \pm 0.009$ & $0.163 \pm 0.022$ & $0.220 \pm 0.098$ & $0.173 \pm 0.011$ & $0.193 \pm 0.007$ & $0.188 \pm 0.010$ \\
\hline Water content $(\%)$ & $58.8 \pm 1.1$ & $59.7 \pm 4.1$ & $69.6 \pm 0.7$ & $65.9 \pm 0.1$ & $69.3 \pm 1.4$ & $67.3 \pm 0.6$ & $76.0 \pm 1.8$ & $69.6 \pm 2.4$ \\
\hline Porosity (\%) & $53.4 \pm 4.1$ & $58.6 \pm 8.5$ & $64.6 \pm 6.0$ & $63.8 \pm 7.7$ & $68.7 \pm 8.6$ & $69.6 \pm 3.4$ & $67.5 \pm 6.5$ & $70.5 \pm 2.1$ \\
\hline Tensile strength (kg) & $3.9 \pm 1.1$ & $4.0 \pm 1.1$ & $6.0 \pm 1.0$ & $5.0 \pm 1.4$ & $8.2 \pm 1.3$ & $8.6 \pm 1.2$ & $3.7 \pm 1.3$ & $4.7 \pm 0.9$ \\
\hline Contact angle $\left({ }^{\circ}\right)$ & $39.1 \pm 2.2$ & $34.4 \pm 4.0$ & $38.5 \pm 1.8$ & $39.2 \pm 3.6$ & $43.5 \pm 2.0$ & $43.6 \pm 1.4$ & $50.3 \pm 3.3$ & $50.4 \pm 4.3$ \\
\hline Hexane (24 h) & - & - & - & - & - & - & - & - \\
\hline DCM (24 h) & + & + & + & + & + & + & + & + \\
\hline DCM/Hexane (24 h) & - & - & - & - & - & - & + & + \\
\hline
\end{tabular}
strength and elongation at break) of the selected membranes

Table 3 Physicochemical and mechanical properties of polymeric membranes a)

a) "_" not dissolved; "+" dissolved. 
are shown in Table 3. The tensile strength of the CA polymer membrane was lower than those of the CTA, CA-CTA, and $\mathrm{CAB}$ membranes; the order of their tensile strengths was $\mathrm{CAB}<\mathrm{CA}<\mathrm{CA}-\mathrm{CTA}<\mathrm{CTA}$. Triolein-embedded CTA (TECTA) had a higher tensile strength, $8.6 \mathrm{~kg}$, than those of the other triolein-embedded passive samplers; this value is 2.2 times that of TECAM, 1.7 times that of TECA-CTA, and 1.8 times that of TECAB. The elongation at break values of the membranes also showed the same order, with TECTA showing a higher value $(27.3 \%)$ than the other membranes. These results indicated that TECTA membranes have better mechanical properties than those of the other membranes and are more suitable for use in turbulent aquatic environments.

Different materials showed different swelling patterns in solvents. The results for solvent-polymer compatibility showed no apparent impact in hexane and dissolution in DCM of all the membrane sheets (Table 3). All the test materials retained their original shape in hexane/DCM $(\mathrm{v}: \mathrm{v}$, $1: 1$ ), except for CAB, which dissolved in this solvent. This indicated that the selected materials swelled little in this solvent, making this an attractive solvent for the extraction of target compounds from the samplers after exposure. Obviously, polymers that are soluble in organic solvents are not suitable for passive sampler construction. Solvents may swell the structure and change the properties of polymers [11]. Extensive swelling makes a polymer fragile, making it difficult to handle.

\subsection{Hydrophilicity of polymer membranes}

The hydrophilicity of the membranes was studied based on the contact angle; the results are shown in Table 3. The contact angles of the membranes were all lower than $90^{\circ}$, indicating that they were hydrophilic and likely to absorb water. Significant differences among the values of the contact angles of the membranes were found. The values of the contact angles of the membranes were in the following order: $\mathrm{CAB}>\mathrm{CTA}>\mathrm{CA}>\mathrm{CA}-\mathrm{CTA}$, which showed that the $\mathrm{CA}$ membrane is more hydrophilic than the CTA and CAB membranes, and that addition of a small amount of triolein to the cast solution did not significantly change the membrane hydrophilicity. For applications in passive sampling materials, membrane hydrophilicity should be taken into account. Some studies have shown that using hydrophilic membranes for sampling hydrophobic compounds results in enhanced transport resistance by the membrane and reduced sampling rates. For example, the uptake kinetics of hydrophobic compounds by membrane-enclosed sorptive coating and Chemcatcher were greatly enhanced by replacing the hydrophilic membrane by polyethylene [22]. It has been reported that the uptake rates of solvent-containing LDPE bags were 24 to 48 times higher than those of solvent-containing cellulose bags [12], but cyclohexane loss was much greater from LDPE bags. This indicated that the cellulose dialysis membranes had much greater resistance to hydrophobic compound transfer than did LDPE membranes. In an effort to optimize the triolein-containing passive sampler design, we developed a novel passive sampler with a TECAM [15]. The uptake rates of PAHs in a TECAM were higher than those in an SPMD (LDPE membrane) [16]. This finding indicated that a hydrophilic CA membrane has an advantage over LDPE. An SPMD/TECAM combination was proposed by Liao et al. [17]. This device, called a TCAPE, consists of an LDPE membrane and an external CA side, and is filled with triolein. Hydrophobic OCPs are accumulated more quickly by TCAPEs than by standard SPMDs. This finding confirmed that a hydrophilic CA membrane has a higher uptake rate-constant than an LDPE does. Similarly, in this study, we found that the uptake rate-constants of CA and CTA were higher than that of $\mathrm{CAB}$, which has a higher contact angle.

\subsection{Uptake kinetics of OCPs by different passive samplers}

Uptake curves (accumulated analyte against time of exposure) for the six test compounds with different triolein- embedded membrane passive samplers are plotted in Figure 1. The concentrations of OCPs in the triolein-embedded membrane passive samplers were proportional to exposure time, with coefficients higher than 0.9 (except TECAB, whose coefficients ranged from 0.68 to 0.81 ). The triolein-embedded passive devices with CA, CA-CTA, and CTA membranes showed rapid uptake of all the OCPs; similar uptake patterns were obtained with these three membranes. TECAB showed only limited accumulation over this period, showing that it is unsuitable as a passive sampler. The uptake rateconstants for TECTA were higher than those for TECAM; the values of $k_{\mathrm{u}}$ calculated using eq. (2) show that the rates of OCPs uptake in TECTA were 1.04-1.43 times higher than those in TECAM. For optimum sampler performance, high uptake rates are essential, especially for accumulation of non-polar chemicals because of their extremely low concentrations in water environments.

\subsection{Effects of triolein on uptake performance}

"Is an adsorbent indispensable in the use of passive samplers?" This is one of the most frequently asked questions about the use of passive samplers. Some authors have therefore studied pollutant uptake behavior using only single-phase polymeric materials such as LDPE [23], polyoxymethylene [24], and silicone rubber as passive samplers [25]. Such passive samplers are cheaper and the sample processing procedure is much simpler compared with conventional SPMDs. Pure triolein, which is expensive, is used as the receiving phase in SPMDs and TECAMs. In this study, a comparison of OCPs uptake in CA, CTA strips, TECAM, and TECTA were performed. The results showed that addition of a small amount of triolein to the membrane 

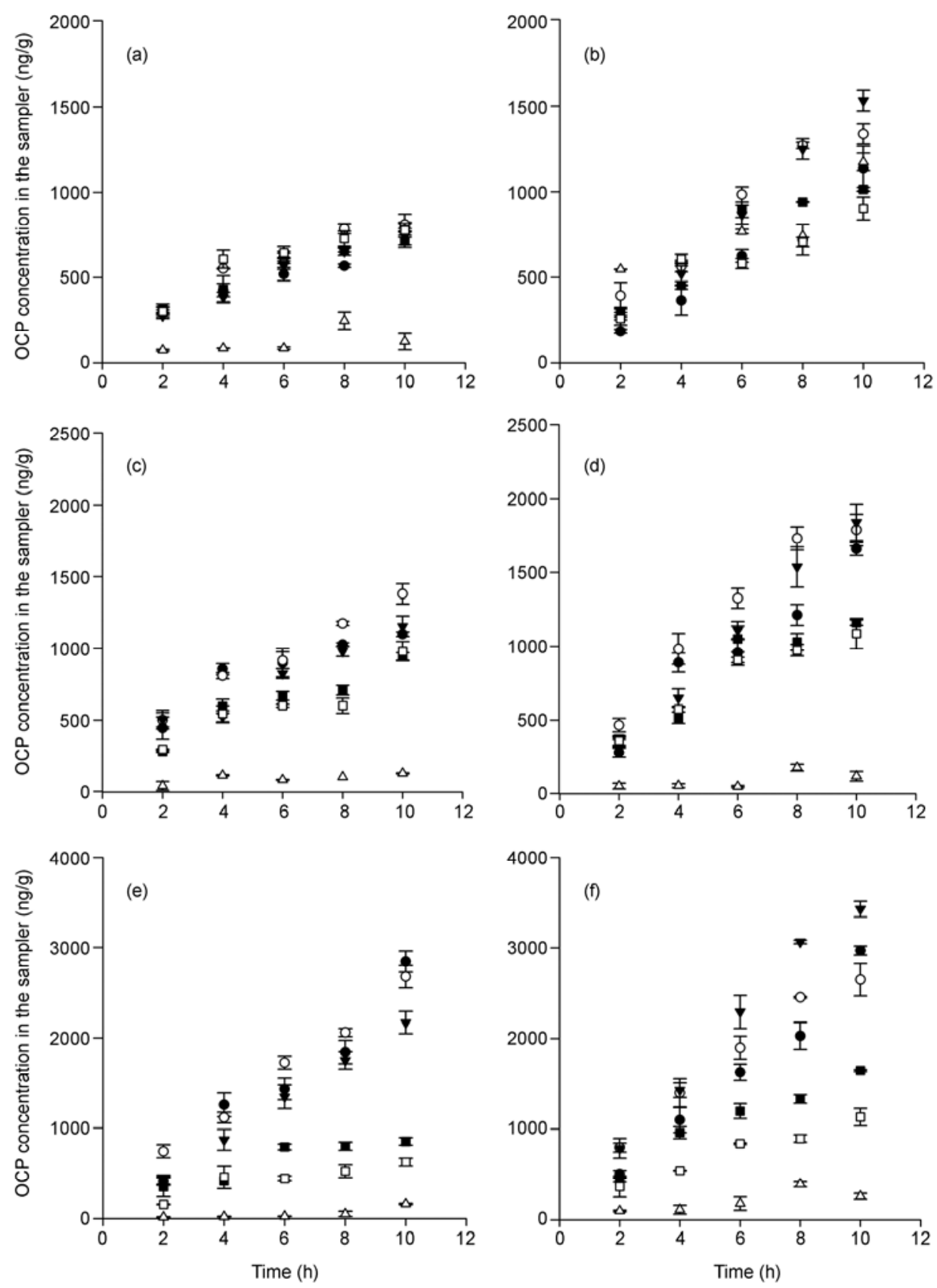

Figure 1 Uptakes of six OCPs, (a) $\gamma$-HCH, (b) aldrin, (c) $\beta$-HCH, (d) heptachlor epoxide, (e) $p, p^{\prime}$-DDE, and (f) endrin, by different polymeric membranes. $\bullet$, TECAM;, TECTA; $\mathbf{v}$, TECA-CTA; $\triangle$, TECAB; $\mathbf{\bullet}$ CA; and $\square$, CTA.

significantly improves the capacity of the samplers to accumulate target chemicals (Figure 1). The uptake rate-constants $k_{\mathrm{u}}$ of CA and CTA were $0.2-1.0$ and $0.2-0.9$ times of those in TECAM and TECTA, respectively (Figure 2). No significant difference was observed between the uptake rate-constant of $\gamma$-HCH $\left(\log K_{\mathrm{ow}}=3.71\right)$ in $\mathrm{CA}$ and that in TECAM, but for high $\log K_{\text {ow }}$ compounds such as $p, p^{\prime}$-DDE $\left(\log K_{\text {ow }}=6.14\right)$, large differences were found; the uptake rate-constants with $\mathrm{CA}$ were significantly lower than those with TECAM (Figure 2) (for $p, p^{\prime}$-DDE, the $k_{\mathrm{u}}$ value in CA was only 0.2 times that in TECAM).
Most lipid-containing passive samplers suffer from interference from lipid impurities, and also need complicated pretreatments. These are reasons for using single-phase passive samplers. Because of their simple construction and low cost, single-phase passive samplers are becoming popular for the sampling of hydrophobic chemicals. However, their drawbacks are that solvent extraction of these polymers extracts oligomers and other co-extracts, which can interfere with instrumental analysis, and their sorption capacities are smaller. In this study, CA and CTA were used as passive samplers, and when they were compared with TECAM, 


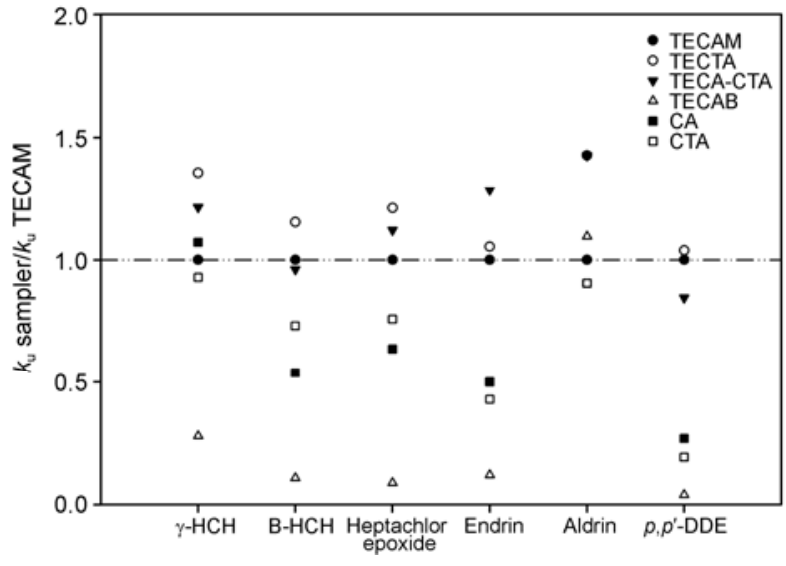

Figure 2 Uptake rate-constants $\left(k_{\mathrm{u}}\right)$ of six OCPs by different passive samplers.

TECTA, TECAM, and TECTA, they showed higher sorption capacities and higher uptake rate-constants, especially for high $\log K_{\text {ow }}$ compounds. Other lipid-containing passive samplers need complicated extraction procedure to remove triolein, but the extraction procedure for TECAM (TECACTA and TECTA) is very simple and uses simple organic solvents. Our results confirmed that it is appropriate to use TECAM or TECTA, rather than CA or CTA, as passive samplers.

\section{Conclusion}

In the present study we evaluated some critical properties of four cellulose-based membranes, i.e., CA, CAB, CA-CTA, and CTA, for use as semipermeable membrane in trioleinembedded membrane passive samplers. In this study, the CTA membrane was found to be the most durable and resistant, with high tensile strength. When triolein was embedded in this membrane, it showed a higher capacity for accumulation than TECAM did. This showed that TECTA is more suitable than TECAM as a passive sampler. On the other hand, $\mathrm{CAB}$ is not a suitable membrane material because of its poor chemical and mechanical resistances and its low uptake of chemicals. Comparisons of CA, CTA, TECAM, and TECTA as passive samplers showed that triolein embedded composite membranes (TECAM and TECTA) are more suitable than single phase membranes ( $\mathrm{CA}$ and CTA).

For further applications of triolein-embedded composite membrane passive samplers, other aspects that were not studied in this paper should be taken into account, such as susceptibility to biofouling and biodegradability of the membrane material with time.

This work was supported by the National Science and Technology Major Project of China (2009ZX07527-005), the National Natural Science Foundation of China (40801204), and the State Key Laboratory of
Environmental Aquatic Chemistry of China (10Y03ESPCR).

1 Huckins J N, Manuweera G K, Petty J D, et al. Lipid-containing semipermeable-membrane devices for monitoring organic contaminants in water. Environ Sci Technol, 1993, 27: 2489-2496

2 Kingston J K, Greenwood R, Mills G A, et al. Development of a novel passive sampling system for the time-averaged measurement of a range of organic pollutants in aquatic environments. J Environ Monitor, 2000, 2: 487-495

3 Vrana B, Popp P, Paschke A, et al. Membrane-enclosed sorptive coating. An integrative passive sampler for monitoring organic contaminants in water. Anal Chem, 2001, 73: 5191-5200

4 Alvarez D A, Petty J D, Huckins J N, et al. Development of a passive, in situ, integrative sampler for hydrophilic organic contaminants in aquatic environments. Environ Toxicol Chem, 2004, 23: 1640-1648

5 Stuer-Lauridsen F. Review of passive accumulation devices for monitoring organic micropollutants in the aquatic environment. Environ Pollut, 2005, 136: 503-524

6 Gorecki T, Seethapathy S, Li X J. Passive sampling in environmental analysis. J Chromatogr A, 2008, 1184: 234-253

7 Soderstrom H, Lindberg R H, Fick J. Strategies for monitoring the emerging polar organic contaminants in water with emphasis on integrative passive sampling. J Chromatogr A, 2009, 1216: 623-630

8 Valor I, Llorca J, Gutierrez C, et al. Constantly stirred sorbent and continuous flow integrative sampler new integrative samplers for the time weighted average water monitoring. J Chromatogr A, 2009, 1216: 5783-5792

9 Allan I J, Booij K, Paschke A, et al. Field performance of seven passive sampling devices for monitoring of hydrophobic substances. Environ Sci Technol, 2009, 43: 5383-5390

10 Allan I J, Harman C, Kringstad A, et al. Effect of sampler material on the uptake of PAHs into passive sampling devices. Chemosphere, 2010, 79: 470-475

11 Rusina T P, Smedes F, Klanova J, et al. Polymer selection for passive sampling: A comparison of critical properties. Chemosphere, 2007, 68: 1344-1351

12 Sabaliunas D, Sodergren A. Uptake of organochlorine pesticides by solvent-filled cellulose and polyethylene membranes. Ecotoxicol Environ Saf, 1996, 35: 150-155

13 Greenwood G M R, Vrana B. Paasive Sampling Techniques in Environmental Monitoring. Amsterdam: Elsevier Press, 2007

14 Huckins J N, Petty J N, Booij K. Monitoring of Organic Contaminants in the Environment: Semipermeable Membrane Devices. New York: Spinger Verlag Press, 2006

$15 \mathrm{Xu} \mathrm{Y,} \mathrm{Wang} \mathrm{Z,} \mathrm{Ke} \mathrm{R,} \mathrm{et} \mathrm{al.} \mathrm{Accumulation} \mathrm{of} \mathrm{organochlorine} \mathrm{pesti-}$ cides from water using triolein embedded cellulose acetate membranes. Environ Sci Technol, 2005, 39: 1152-1157

16 Ke R H, Xu Y P, Wang Z J, et al. Estimation of the uptake rate constants for polycyclic aromatic hydrocarbons accumulated by semipermeable membrane devices and triolein-embedded cellulose acetate membranes. Environ Sci Technol, 2006, 40: 3906-3911

17 Liao L B, Xiao X M. Accumulation of organochlorine pesticides by semipermeable membrane devices using composite complex. Chemosphere, 2006, 64: 1592-1600

18 Vasarhelyi K, Ronner J A, Mulder M H V, et al. Development of wet dry reversible reverse-osmosis membranes with high-performance from cellulose-acetate and cellulose triacetate blends. Desalination, 1987, 61: 211-235

19 KastelanKunst L, Dananic V, Kunst B, et al. Preparation and porosity of cellulose triacetate reverse osmosis membranes. J Membrane Sci, 1996, 109: 223-230

20 Stamatialis D F, Dias C R, de Pinho M N. Atomic force microscopy of dense and asymmetric cellulose-based membranes. J Membrane Sci, 1999, 160: 235-242

21 Ke R H, Luo J P, Sun L W, et al. Predicting bioavailability and accumulation of organochlorine pesticides by Japanese medaka in the presence of humic acid and natural organic matter using passive sampling membranes. Environ Sci Technol, 2007, 41: 6698-6703 
22 Vrana B, Mills G, Greenwood R, et al. Performance optimisation of a passive sampler for monitoring hydrophobic organic pollutants in water. J Environ Monitor, 2005, 7: 612-620

23 Booij K, Smedes F, van Weerlee E M. Spiking of performance reference compounds in low density polyethylene and silicone passive water samplers. Chemosphere, 2002, 46: 1157-1161

24 Janssen E M L, Oen A M P, Luoma S N, et al. Assessment of field-related influences on polychlorinated biphenyl exposures and sorbent amendment using polychaete bioassays and passive sampler measurements. Environ Toxicol Chem, 2011, 30: 173-180

25 Rusina T P, Smedes F, Koblizkova M, et al. Calibration of silicone rubber passive samplers: Experimental and modeled relations between sampling rate and compound properties. Environ Sci Technol, 2010, 44: 362-367

Open Access This article is distributed under the terms of the Creative Commons Attribution License which permits any use, distribution, and reproduction in any medium, provided the original author(s) and source are credited. 